\title{
Téoros
}

Revue de recherche en tourisme

\section{Le patrimoine industriel ...à la carte}

\section{Marie-Claude Robert}

Volume 15, numéro 2, été 1996

Patrimoine industriel

URI : https://id.erudit.org/iderudit/1075031ar

DOI : https://doi.org/10.7202/1075031ar

Aller au sommaire du numéro

Éditeur(s)

Université du Québec à Montréal

ISSN

0712-8657 (imprimé)

1923-2705 (numérique)

Découvrir la revue

Citer ce document

Robert, M.-C. (1996). Le patrimoine industriel ...à la carte. Téoros, 15(2), 55-56. https://doi.org/10.7202/1075031ar d'utilisation que vous pouvez consulter en ligne.

https://apropos.erudit.org/fr/usagers/politique-dutilisation/ 


\section{LE PATRIMOINE INDUSTRIEL ...À LA CARTE}

Marie-Claude Robert, présidente Association québécoise pour le patrimoine industriel

L'Association quebécoise pour le patrinoine industriel a ête fondec en 1988 . Elle se donne la mission de promouvoir la mise en valeur du patrinoine industriel el de favoriser la connaissance dans ce domaine. La diffusion par le biais d'événements et de publications complète la sphère de ses activites. Sans âtre directement lice au doveloppement du tourisme industriel, elle y contribue en soufenant la réflexion de ses membres par la tenue de ses congrès thématiques et les voyages d'étude qu'elle organise ici comme d' l'etranger.

L'Aqpi s'est doté d'outils precieux pour la mise en valeur du patrimoine industriel. Elle leur a donné la forme d'un Répertoire des intervenants en patrinoine industriel, d'une Bibliographie du patrimoine industriel et d'un Guide d'archives sur le patrinoine industriel.

Après avoir consolidé sa structure et gagné la reconnaissance des experts, organtismes et promoteurs actifs en patrinoine industriel. I'Aapi élargit progressiventent son champ d'action vers un public plus large. Cette étape est en quelque sorte le debouche naturel du travail de mise en place d'un réseau et de recherchesà valeur scientifique qui ont été essentielles à la constitution de l'orgamisnte.

En entreprenant son plus récent projet de Cartographie des sites industriels d'intérêt patrimonial au Québec, l'Aqpi voulait, en prenter lieu, reperer son "terrain de chasse" et ainsi mieux lire l'ampleur potentielle de la mise en valeur du parinoine industriel au Québec. Cette démarche révele parallèenent l"oppontunité de créer un outil de promotion auprès d'une clientèle touristigue tout autant qu'auprès du milieu industriel qui serait en mesure de l'accueillir.

\section{UN SECOND REGARD SUR L'INDUSTRIE}

Après plus d'une génération de mutation économique, sociale et environnementale qui nous laisse troublés et songeurs, notre collectivité, comme bien d'autres avec elle, sent le besoin de renouer avec la vie industrielle et la tradition dont elle est porteuse. C'est dans sa dimension cul- turelle que l'activité industrielle rejoint le plus large public et détient une valeur touristique qui peut $\mathrm{s}^{7} \mathrm{y}$ associer. $\mathrm{Ce}$ tourisme à saveur historique oflie un acces privilegié à la compréhension du développement du territoire et de son peuplement. Par ailleurs, il ne faut pas oublier que le milieu indusuriel est funilier pour une vaste couche de la population qui y travaillait encore récomment, ou $l^{*}$ a côtoyé dans son environnement quotidien. Pour sa part, le milieu industriel actif semble encore assez loin de telles préoccupations, sa tâche en étant une de production. Ce patrimoine lui suggère un regard en arrière et contribue à la continuite $d^{4} u n e$ culture et d'une entreprise. Nous sommes à tracer le chemin qui permettra de $s^{*} a l l i e r$ la participation nécessaire du milieu industriel à la mise en valeur de notre patrimoine commun. C'est dans cette perspective que l'Aqpi examine les pistes qu'elle aura à explorer dans son projet de cartographie des sites industriels d'intérêt patrimonial.

\section{SUR LE CHEMIN DE L'USINE}

Tout d'abord, l'inventaire, initiế par l'Aqpi avec le support du ministère de la Culture et des Communications, a révélé d'intéressantes surprises. La recherche effectuée par madame Nicole Dorion a permis d'identifier près de 1000 entreprises (le nombre est presque déconcertant) encore actives au Québec et fondées avant 1950 (date fixee pour établir $\mathrm{l}^{+}$intérêt patrimonial). Un bassin considérable, donc, qui se réduira largement lorsque seront connues les données sur la structure d'accueil des visiteurs. A ce nombre, viendront s'ajouter les sites indusuriels ayant cessé leurs activités mais êtant intếgrés à un circuit ou un centre permettant leur interprétation.

Le projet de cartographie a pour objectif de proposer au visiteur (vacancier, scolaire, professionnel et autres) la découverie entre autres de la vie "industrieuse", des développements économiques, des 
modes de vie, et du développement des technologies, de la région touristique qu'il explore. Elle permet en outre de rendre compte des efforts consentis à la mise en valeur du patrimoine industriel de chaque région du Québec. La carte sera complétée d'ici un an et fera l'objet d'une diffusion élargie. Sa publication impliquera également la participation financière des entreprises identifiées.

Il est vite apparu que le travail d'inventaire offre d'autres débouchés intêressants. L'association y voit une possibilité de poursuivre sa mission en investiguant avec ces entreprises l'approche la plus apte à les associer à la mise en valeur de leur patrimoine et à favoriser la création d'activités ou la diffusion de matériel d'interprétation. Il semble stratégique de susciter des maintenant ce rapprochement avec un milieu industriel encore bien vivant afin de conjuguer le patrimoine industriel à sa valeur de transmission plutôt qu'à sa menace de disparition.

\section{QUEL PATRIMOINE INDUSTRIEL}

Il est certain que ces entreprises ont dû évoluer et ajuster leur technologie, leurs infrastructures, voire leur production pour se maintenir actives jusqu'à ce jour. C'est ce qui sera révélé par la recherche au cas par cas de leur richesse patrimoniale.

La mise en valeur du patrimoine industriel s'applique à trois types de situation. Nous y trouvons les périodes industrielles dont les acteurs ont disparu en nous laissant le témoignage de leurs vestiges ou de leurs archives. Dans ce cas, c'est comme société que nous jugeons de sa valeur et faisons l'effort d'en préserver des traces matérielles. Le résultat est un réseau de sites et centres d'interprétation témoignant d'une étape industrielle de l'histoire d"une région.

Il y a un certain nombre d'entreprises dont $l^{\prime}$ envergure régionale ou nationale dominante commande pour divers facteurs (dont certains sont plus nobles que d'autres) une démarche de mise en valeur de leur patrimoine industriel associe a leur importance stratégique dans 1'histoire de la communauté. Ces grandes entreprises sont bien présentes dans le

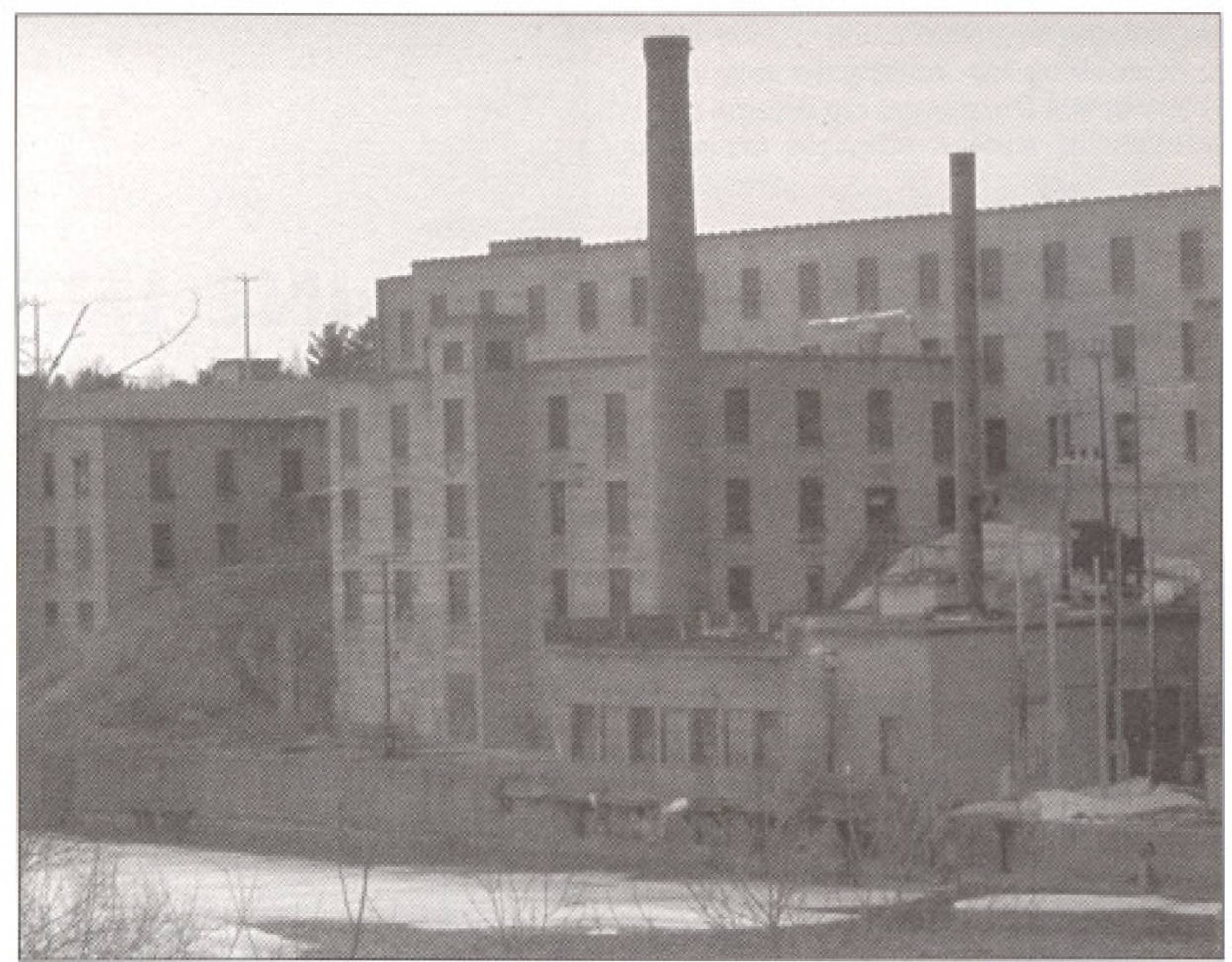

Bâtinents industriels à Lachute. Le Quebec compte un grand nombre de villes industrielles construites atutour d'un pouwoir hydratique.

panorama du tourisme industriel où elles assument la transmission de leur patrimoine.

Il existe enfin une troisième catégorie dont l'invetaire des sites nous révèle l'ampleur. Ce sont les entreprises qui tissent le réseau industriel de nos régions mais dont l'attention, on peut le supposer à juste titre, porte plus sur le potentiel de développement que sur la valeur de leur patrimoine. Il s'agit bien là d'un potentiel touristique que chaque communauté peut contribuer à développer.

En fait, le projet de cartographie nous permet d'anorcer une action plus systématique auprès des entreprises actives pour y susciter tout d'abord un intérêt et une rếtlexion à l'égard de leur patrimoine et oeuvrer de concert avec elles à sa diffusion. Ce patrimoine bien vivant, nous le connaissons assez mal. Il n'y avait pas "péril en la demeure" et nos industriels ont inscrit leur destinée présente dans la foulée de leur passé. Qu'en ont-ils conservé? Quelle valeur lui attribuent-ils? Ces données-là nous manquent et c'est un champ immense à couvrir.

En tentant de rejoindre les industriels sur leur terrain, ce projet convie les acteurs du patrimoine industriel à poursuivre le rêve qui émerge de toute réflexion sur le patrimoine: c'est qu'il soit porté par ceux-là même qui le produisent. Plus réalistement, il est dans notre propos de valoriser auprès de notre milieu industriel la transmission de son apport patrimonial au sein de la communauté. 